\title{
Off-design Performance Analysis of Multi-Stage Transonic Axial Compressors
}

\author{
W. H. Du, H. Wu, L. Zhang \\ School of Power and Energy Northwestern Polytechnical University, Xi'an 710072, China \\ Email: wenhai.du@gmail.com
}

\begin{abstract}
Because of the complex flow fields and component interaction in modern gas turbine engines, they require extensive experiment to validate performance and stability. The experiment process can become expensive and complex. Modeling and simulation of gas turbine engines are way to reduce experiment costs, provide fidelity and enhance the quality of essential experiment. The flow field of a transonic compressor contains all the flow aspects, which are difficult to present-boundary layer transition and separation, shock-boundary layer interactions, and large flow unsteadiness. Accurate transonic axial compressor off-design performance prediction is especially difficult, due in large part to three-dimensional blade design and the resulting flow field. Although recent advancements in computer capacity have brought computational fluid dynamics to forefront of turbomachinery design and analysis, the grid and turbulence model still limit Reynolds-average Navier-Stokes (RANS) approximations in the multi-stage transonic axial compressor flow field. Streamline curvature methods are still the dominant numerical approach as an important tool for turbomachinery to analyze and design, and it is generally accepted that streamline curvature solution techniques will provide satisfactory flow prediction as long as the losses, deviation and blockage are accurately predicted.

An improved streamline curvature numerical method is developed and applied to better approximate the flow fields of multi-stage transonic axial compressor. Specifically, improvements in total pressure loss modeling are implemented to ensure accurate and reliable off-design performance prediction. Compared with the original normal shock, the new shock loss model accounts for shock geometry changes, with shock loss estimated as a function of inlet relative Mach number, flow turning, solidity, leading edge radius and suction surface profile from near-choke to near-stall. The importance of accounting for shock geometry and loss changes with operating conditions is clearly demonstrated. The 3-D effects on the deviation and loading effects on the tip secondary loss are also accounted in this paper.

The data from a two-stage transonic axial compressor (NASA TP-1493) provides the basis for experimental comparisons. In general, the effects of important flow phenomena relative to off-design performance of the transonic compressor are adequately captured, which include the shock loss, second flow and boundary layer transition. The increased shock pressure loss with loading across the supersonic first-rotor tip region indicates that the new shock model can be considered a significant improvement in the analysis of multi-stage transonic compressor. Overall performance and spanwise: profile comparisons demonstrate that the improved model gives reasonable performance trends and generally accurate results, indicating that the physics-based shock loss model is correct and applicable.
\end{abstract}

